\title{
MECHANICAL RESPONSE OF ZIRCONIUM-I. DERIVATION OF A POLYCRYSTAL CONSTITUTIVE LAW AND FINITE ELEMENT ANALYSIS
}

\author{
C. N. TOMÉ ${ }^{1} \dagger$, P. J. MAUDLIN ${ }^{1}$, R. A. LEBENSOHN ${ }^{2}$ and G. C. KASCHNER ${ }^{1}$ \\ ${ }^{1}$ Los Alamos National Laboratory, MST-8 MS G755, Los Alamos, NM 87545, USA and ${ }^{2}$ Instituto de \\ Física Rosario (UNR-CONICET), 2000 Rosario, Argentina
}

( Received 13 December 2000; received in revised form 24 April 2001; accepted 24 April 2001 )

\begin{abstract}
Simulating the forming of anisotropic polycrystals, such as zirconium, requires a description of the anisotropy of the aggregate and the single crystal, and also of their evolution with deformation (texture development and hardening). Introducing the anisotropy of the single crystal requires the use of polycrystal models that account for inhomogeneous deformation depending on grain orientation. In particular, viscoplastic self-consistent models have been successfully used for describing strongly anisotropic aggregates. As a consequence, using a polycrystal constitutive law inside finite element (FE) codes represents a considerable improvement over using empirical constitutive laws, since the former provides a physically based description of anisotropy and its evolution.

In this work we develop a polycrystal constitutive description for pure $\mathrm{Zr}$ deforming under quasi-static conditions at room and liquid nitrogen temperatures. We use tensile and compressive experimental data obtained from a clock-rolled $\mathrm{Zr}$ sheet to adjust the constitutive parameters of the polycrystal model. Twinning is accounted for in the description. The polycrystal model is implemented into an explicit FE code, assuming a full polycrystal at the position of each integration point. The orientation and hardening of the individual grains associated with each element is updated as deformation proceeds. We report preliminary results of this methodology applied to simulate the three-dimensional deformation of zirconium bars deforming under four-point bend conditions to maximum strains of about $20 \%$. A critical comparison between experiments and predictions is done in a second paper (Kaschner et al., Acta mater. 2001, 49(15), 3097-3107). Published by Elsevier Science Ltd on behalf of Acta Materialia Inc.
\end{abstract}

Keywords: Texture; Polycrystal modeling

\section{INTRODUCTION}

Simulating the forming of anisotropic polycrystals, such as zirconium, requires a description of the anisotropy of the aggregate and the single crystal, and also of their evolution with deformation (texture development and hardening). The anisotropy of the single crystal requires the use of polycrystalline models that account for inhomogeneous deformation depending on the grain orientation. Self-consistent visco-plastic models have been successfully used for describing strongly anisotropic metallic and geological aggregates [1-5] and will be used in what follows for describing the constitutive response of pure $\mathrm{Zr}$ with a strong initial texture. The ultimate goal of this study is to provide a realistic description of the constitutive response of the material for use in finite element (FE) calculations which fully account for mechanical anisotropy, hardening and twinning

\footnotetext{
$\dagger$ To whom all correspondence should be addressed E-mail address: tome@lanl.gov (C. N. Tomé)
}

mechanisms, and their evolution with deformation as well.

This work has three components which illustrate the paradigm associated with simulating forming operations: a robust constitutive description of the mechanical response; a comprehensive experimental characterization of the system; and accurate FE techniques for dealing with non-uniform deformation associated with complex boundary conditions. We use experimental information (texture, loading curves, metallographic evidence) to infer the characteristics of the active deformation modes and their interaction (hardening) in $\mathrm{Zr}$ at room temperature (RT) and liquid nitrogen temperature (LNT). We use for such purpose a visco-plastic self-consistent (VPSC) polycrystal model [1] in which we incorporate an improved treatment of twinning. Secondly, we implement the polycrystal description into the explicit FE code EPIC-97 [6], and apply this methodology to predict the deformation of a rectangular $\mathrm{Zr}$ bar subjected to a four-point bend test. A polycrystalline aggregate is associated with each FE integration 
point. The FE code imposes the computed velocity gradient on the polycrystal, updates the orientation and the hardening of the individual grains depending on the deformation history of the element, and predicts the macroscopic stress for use in the solution of the continuum equilibrium equations. The advantage of this approach is that it accurately accounts for material anisotropy and its evolution with texture development. The disadvantage is that the calculations are computationally intensive, although feasible on modern computers for problems not requiring fine spatial resolution.

\section{MATERIAL AND EXPERIMENTS}

The material in this study is crystal-bar zirconium, purchased from Teledyne Wah Chang, processed via a series of clock rolling and vacuum annealing cycles to produce a plate with strong basal texture and approximate in-plane axisymmetry (Fig. 1). The aggregate exhibits equiaxed grains with a mean size of ca $25 \mu \mathrm{m}$. Results of compression tests done in cylindrical specimens cut from the plate and tested in the through-thickness (TT) and the in-plane (IP) directions have been reported by Kaschner et al. [7] and Kaschner and Gray [8], who studied the effects of texture and interstitial impurities.

In this paper we use the experimental compression test data to adjust the constitutive response of highpurity zirconium. In addition, we present new results of tensile tests meant to provide information on the asymmetric character of the mechanical response of Zr. Cylindrical tensile specimens with a nominal central gauge of $17.7 \mathrm{~mm}$ length and $2.25 \mathrm{~mm}$ diameter were machined with their axes parallel to the plane of the plate. Mechanical tests were performed at 76 and $293 \mathrm{~K}$ at a strain rate of $0.001 \mathrm{~s}^{-1}$ to an accumulated plastic strain of ca $25 \%$ along the testing direction. The tensile loading curves, together with the compression curves, are depicted in Fig. 2. for both testing temperatures. Notice the striking difference between the TT compression (TTC) and the IP compression (IPC) response at both temperatures. This difference can be explained qualitatively by the fact that the $\mathrm{Zr}$ crystals are hard to deform along the $c$ axis, while they can accommodate deformation by easy prism slip when the testing direction is perpendicular to the $c$-axis. As for comparing specimens tested in-plane, notice the different hardening exhibited by the IPT and the IPC curves. This is especially evident at LNT, where the section aspect ratios of the tensile and compression samples are also very different (see Fig. 3). In what follows we will attribute such response to grain reorientation by tensile twinning during IPC, which leads to a texture "randomization" in the sample, but which also introduces strong barriers to further propagation of dislocations or twins.

\section{POLYCRYSTAL MODEL AND SINGLE CRYSTAL PARAMETERS}

The VPSC polycrystal formulation originally proposed by Molinari et al. [9] and later implemented for anisotropic plasticity by Lebensohn and Tomé [1] is used for the polycrystal analysis presented here. The formulation is briefly reviewed in what follows, while the hardening and twinning models are described in some detail because they are relevant to the interpretation of the experiments. Within the VPSC formulation the polycrystal is represented as an aggregate of orientations with weights that represent volume fractions chosen to reproduce the initial texture. Each grain is treated as an ellipsoidal inclusion embedded in an anisotropic medium. The shear rate in each system is a power of the resolved shear stress divided by a threshold value. The strain rate in the grain is given by the sum over the shears contributed by all systems:

$$
D_{i j}^{\mathrm{c}}=\dot{\gamma}_{0} \sum_{s} m_{i j}^{s}\left(\frac{m^{s}: \sigma^{\prime \mathrm{c}}}{\tau^{s}}\right)^{n}=M_{i j k l}^{\mathrm{c}}\left(\sigma^{\prime}\right){\sigma_{k l}}^{{ }^{\prime}}{ }^{\mathrm{c}}
$$

where $D^{\mathrm{c}}, M^{\mathrm{c}}, \sigma^{\mathrm{c}}, m^{s}$ are the strain rate, the viscoplastic compliance, the deviatoric stress, and the Schmid tensors for the grain. The exponent $n$ is set to $n=20$ in our calculations, and equation (1) has to be interpreted as a strategy for resolving the activity in each system without ambiguity rather than as describing the actual rate sensitivity of the material [11]. The overall response of the homogeneous effective medium is described by a pseudo-linear law relating overall strain-rate and overall stress:

$$
\bar{D}=M^{(\mathrm{sec})}: \bar{\sigma}^{\prime} .
$$

When the stress equilibrium equation is solved for
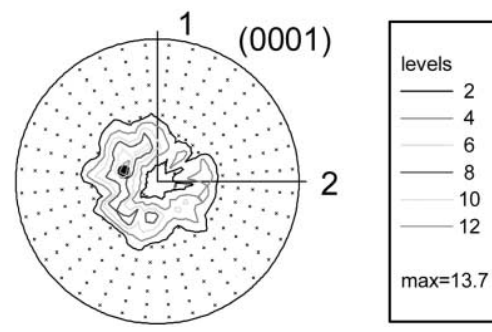
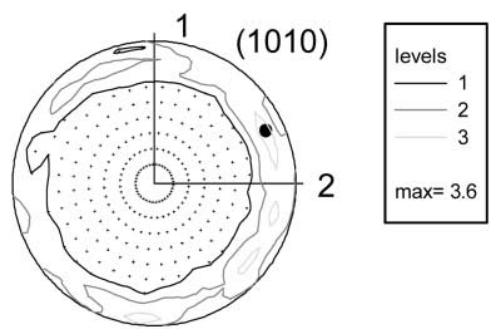

Fig. 1. Initial texture (basal and prism pole figures) of clock rolled $\mathrm{Zr}$ used in this study. Direction 3 coincides with the plate normal (ND). 

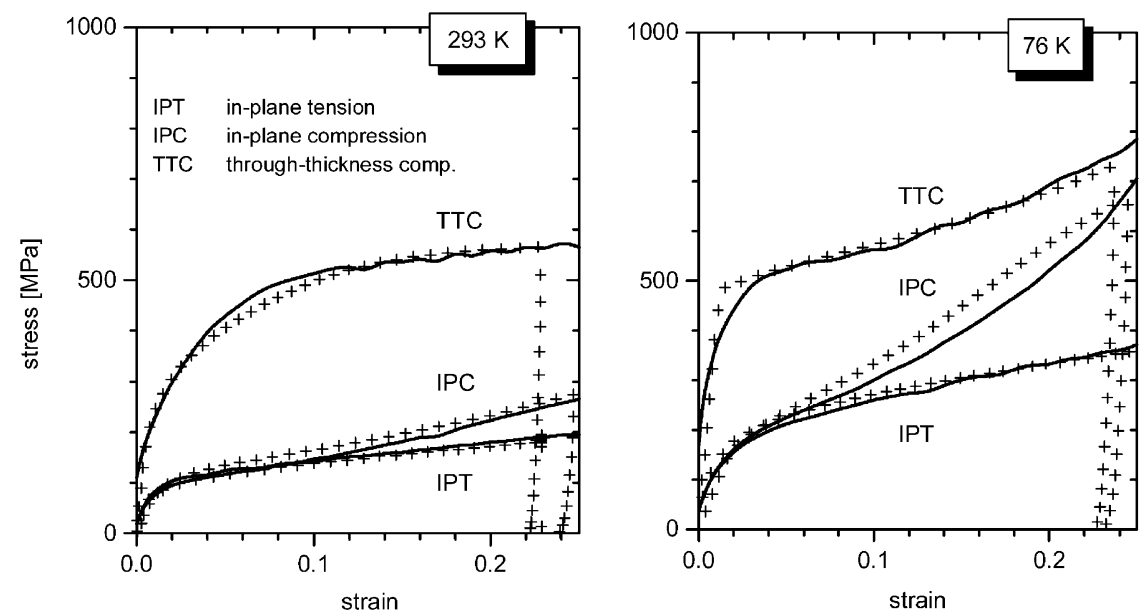

Fig. 2. Experimental (+) and predicted (- loading curves for clock rolled Zr at RT and LNT. Throughthickness compression (TTC), in-plane compression (IPC) and in-plane tension (IPT).

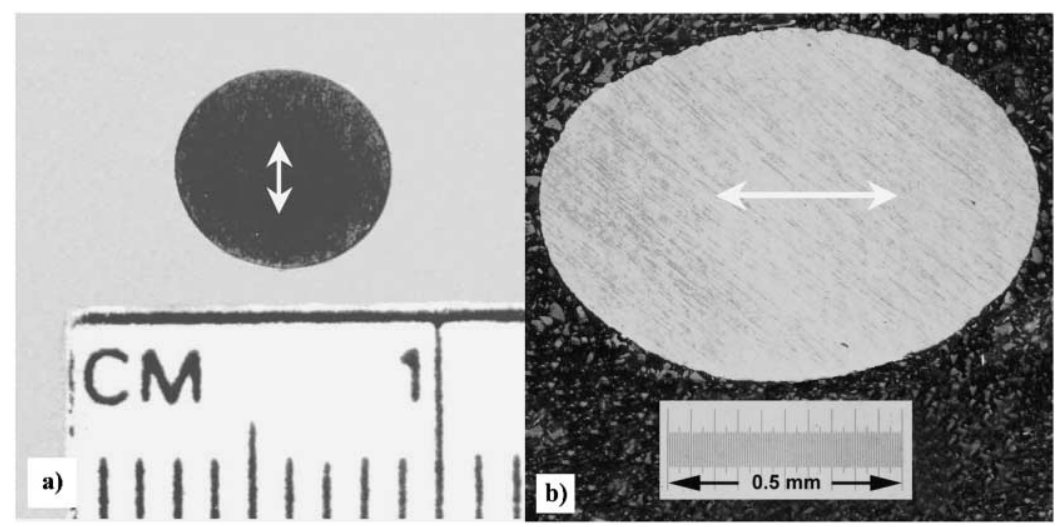

Fig. 3. Cross-section at the midpoint of high-purity zirconium samples deformed at LNT (76K) along the inplane direction. (a) Deformed in compression (IPC) to $24 \%$ true strain; (b) deformed in tension (IPT) to $25 \%$ true strain. Double-ended arrows indicate the initial orientation of the basal poles.

the visco-plastic inclusion the following interaction equation results:

$$
\left(D^{\mathrm{c}}-\bar{D}\right)=-M^{*}:\left(\sigma^{\mathrm{c}}-\bar{\sigma}^{\prime}\right)
$$

where

$$
M^{*}=n^{\mathrm{eff}} M^{(\mathrm{sec})}:(I-E)^{-1}: E
$$

and $E$ is the visco-plastic Eshelby tensor [10]. Observe that we use $n^{\text {eff }}=10$ in the interaction equation and $n=20$ in equation (1). This is done in order to enforce a more rigid interaction than the $n^{\text {eff }}=n$ associated with the tangent formulation [11]. This feature changes the results quantitatively but not qualitatively, and provides grain strain deviations from the average which are intuitively more realistic. Observe, also, that when $n^{\text {eff }} \rightarrow 0$ equation (3) tends to give the Full Constraints (Taylor) case. The condition represented by equation (3) allows the deformation to differ from grain to grain depending on the relative anisotropy between each grain and the surrounding matrix. Typically, grains unfavorably oriented for accommodating an imposed strain will deform less than those favorably oriented.

\subsection{Hardening of slip and twinning systems}

The threshold stress $\tau^{s}$, which appears in equation (1), describes (in an average way) the resistance for activation that the deformation modes experience and it usually increases with deformation due to strainhardening. Here we define a reference hardening function for each system, described by:

$$
\hat{\tau}^{s}=\tau_{0}^{s}+\left(\tau_{1}^{s}+\theta_{1}^{s} \Gamma\right)\left(1-\exp \left(-\frac{\theta_{0}^{s} \Gamma}{\tau_{1}^{s}}\right)\right)
$$

where $\Gamma$ is the accumulated shear in the grain. Equation (4) represents an extended Voce law which, instead of stress saturation, exhibits an asymptotic hardening rate $\theta_{1}^{s}$. While the latter could be regarded as describing stage IV at large strains, for the strains used in this work it is more of an adjustable hardening parameter. In addition, we allow for "self" and "lat- 
ent" hardening by defining coupling coefficients $h^{s s^{\prime}}$ which account for the obstacles that dislocations in system $s^{\prime}$ represent to the propagation of $s$ dislocations. Eventually, the increase in the threshold stress of a system due to shear activity in the grain systems is calculated as:

$$
\Delta \tau^{s}=\frac{\mathrm{d} \hat{\tau}^{s}}{\mathrm{~d} \Gamma} \sum_{s^{\prime}} h^{s s^{\prime}} \Delta \gamma^{s^{\prime}}
$$

when "self" and "latent" hardening are indistinguishable then $h^{s s^{\prime}}=1$ and the evolution of the threshold stress is solely given by the reference hardening function $\hat{\tau}^{s}$

$$
\frac{\Delta \tau^{s}}{\Delta \Gamma}=\frac{\mathrm{d} \hat{\tau}^{s}}{\mathrm{~d} \Gamma} \text { and } \tau_{(\Gamma)}^{s}=\hat{\tau}_{(\Gamma)}^{s}
$$

Equations (4)-(6) permit us to describe the high hardening rate observed at the onset of plasticity, and its decrease towards saturation at large strains. Linear hardening is a limiting case of this law, and takes place when $\tau_{1}^{s}=0$.

\subsection{Twinning model}

We assume that twinning is analogous to slip in that a twin system has a critical resolved shear of activation in the twinning plane and along the twinning direction. However, it differs from slip in its directionality, which we model by allowing shear only in the positive sense of the twin "Burgers" vector. Another aspect of twinning that needs to be incorporated into the models is the fact that the twinned fractions are regions (usually of lamellar or lenticular morphology) with a different orientation than the surrounding matrix. These twinned regions not only contribute to the texture of the aggregate but, more importantly, act as effective barriers for the propagation of dislocations and for the growth of other twin lamellae. The hardening induced by the twins is empirically enforced here by assigning high values to the latent hardening coefficients $h^{s s^{\prime}}$ which describe slip-twin and twin-twin interactions.

As for the effect on texture of the twinned fractions, here we use the predominant twin reorientation scheme proposed by Tomé et al. [12], which works as follows: within each grain $g$, we keep track of the shear strain $\gamma^{t, g}$ and of the associated volume fraction $V^{t, g}=\gamma^{t, g} / S^{t}$ contributed by each twin system $t$ ( $S^{t}$ is the characteristic twin shear). The sum over all twin systems of a certain kind (e.g., tensile twins) and over all grains represents the accumulated twin fraction $V^{\text {acc }}$ in the aggregate (i.e., the volume fraction of tensile twins that one would measure from a micrograph):

$$
V^{\mathrm{acc}}=\sum_{g} \sum_{t} \frac{\gamma^{t, g}}{S^{t}} .
$$

Since it is not numerically feasible (nor physically justifiable) to regard each twinned fraction as an independent new grain, the predominant twin reorientation scheme adopts a statistical approach. At each incremental step some grains are fully reoriented by twinning provided certain conditions are fulfilled. Calling the volume fraction represented by these fully reoriented grains for each of the twin modes the effective twinned fraction ( $V^{\mathrm{eff}}$ ), we define a threshold volume fraction as

$$
V^{\mathrm{th}}=A^{\mathrm{th} 1}+A^{\mathrm{th} 2} \frac{V^{\mathrm{eff}}}{V^{\mathrm{acc}}} .
$$

After each deformation increment we select a grain at random and identify the twin system with the highest accumulated volume fraction in the grain. If the latter is larger than the threshold $V^{\text {th }}$ associated with that twin mode then the whole grain is reoriented by twinning in the predominant system. The process is repeated until either all grains are randomly checked or until the effective twin volume exceeds the accumulated twin volume. In the latter case we stop reorientation by twinning and proceed to the next deformation step. Two things are achieved in this process: (a) only the historically most active twin system in each grain is considered for reorienting the whole grain by twinning; (b) the twinned fraction is kept consistent with the shear activity that the twins contribute to deformation. The algorithm equation (8) prevents grain reorientation by twinning until a threshold value $A^{\text {th1 }}$ is accumulated in any given system (typically $10-25 \%$ of grain volume) and rapidly stabilizes the threshold at a value around $A^{\text {th1 }}+A^{\text {th2 }}$ (typically $50-60 \%$ of grain volume). In this work we have used $A^{\text {th1 }}=0.1$ and $A^{\text {th2 }}=0.5$, and do not allow twin-reoriented grains to undergo a second reorientation by twinning.

\subsection{Single crystal and polycrystal hardening}

The experimental information [8, 13-15] and the evidence that results from comparing predicted and measured deformation textures of $\mathrm{Zr}$ and $\mathrm{Zr}$-alloys [1, 2, 16-18] indicate that the following systems operate in $\mathrm{Zr}$. At RT and LNT prismatic (pr) slip of the type $\{10 \overline{1} 0\}\langle 11 \overline{2} 0\rangle$ is easily activated. Also tensile twinning (ttw) of the type $\{10 \overline{1} 2\}\langle 10 \overline{1} 1\rangle$ is active at these temperatures and, to a lesser extent, tensile twins of the type $\{10 \overline{2} 1\}\langle 11 \overline{2} \overline{6}\rangle$ which are not accounted for in this work. Tensile twinning, however, cannot accommodate compression along the $c$-axis of the crystal. In addition, the difference between the IPT and IPC loading curves (Fig. 2) indicates that another mode has to be active in compression at RT and LNT. The microscopic evidence (Fig. 4 in Ref. [8]) exhibits very little twinning taking place at RT, which suggests that $\{101 \overline{1}\}\langle 11 \overline{2} 3\rangle$ pyramidal (pyr) slip may be active at RT. On the other hand, twinning is plentiful in the micrographs of samples tested at LNT, suggesting that it replaces pyramidal slip as the favored sys- 


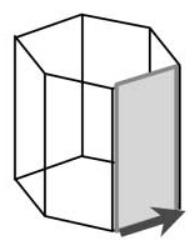

$(1010)<1120>$ prismatic slip

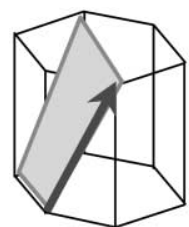

$(1011)<1123>$ pyramidal slip (at $293 \mathrm{~K}$ )

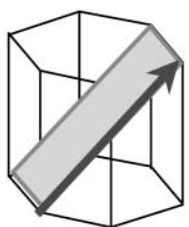

$(1012)<1011>$ tensile twin

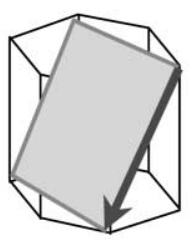

$(1122)<1123>$ compressive twin

(at $76 \mathrm{~K}$ )

Fig. 4. Active deformation systems in $\mathrm{Zr}$ considered in this work: prismatic slip, tensile twinning and pyramidal slip at $293 \mathrm{~K}$; prismatic slip, tensile twinning and compressive twinning at $76 \mathrm{~K}$.

tem. As a consequence, we assume compressive twinning (ctw) of the $\{11 \overline{2} 2\}\langle 11 \overline{2} \overline{3}\rangle$ type at LNT. In what follows we try to find the evolution of the CRSSs associated with the active deformation modes at RT and LNT (Fig. 4). Specifically, we adjust the parameters that appear in the hardening laws equations (4) and (5) until the predicted loading reproduces the experimental response of Fig. 2.

The reference threshold stresses $\hat{\tau}$ that result from such a fitting procedure are plotted in Fig. 5 for each deformation mode and for the two temperatures. At RT, tensile twinning is only slightly harder than prismatic slip, while the fitting indicates that pyramidal slip is much harder. At LNT the threshold stresses for activating prismatic slip and tensile twinning increases, and compressive twinning is favored over pyramidal slip. The parameters associated with the aforementioned modes and temperature regimes are summarized in Table 1. Here we have tried to keep the number of adjustable parameters to a minimum, while aiming for a satisfactory fit of the experimental loading curves (Fig. 2). The initial texture used in the simulation (Fig. 1) consists of 377 discrete orientations with appropriate weights. The number of orientations represents a compromise between accuracy and the running time and RAM requirements of the FE application.

Notice that the reference threshold $\hat{\tau}^{s}$ given by equ- ation (4) and plotted in Fig. 5 does not necessarily describe the actual threshold $\tau^{s}$ for a given system. The actual threshold, which is updated using equation (5), is usually higher because it includes a contribution from the latent hardening coefficients $\left(h^{s s^{\prime}} \geq\right.$ 1). For the fitting procedure we enforce the latent hardening of slip and twinning systems due to slip activity to be the same, namely $h^{s s^{\prime}}=1$. As for the effect of twinning upon slip and the other twinning systems, it is evident from the values of the latent hardening parameters which fit the data, that these interactions are much stronger. The interpretation is that the twin lamellae associated with active twin systems act as barriers for the propagation of dislocations or of other twinning systems. This interpretation is also consistent with the hardening exhibited by the loading curves of Fig. 2: when twinning is active the hardening rate tends to increase past about $10 \%$ deformation as a consequence of these barriers. Observe that, according to Table 1, at LNT prismatic dislocations seem to be strongly impeded by the tensile twins $\left(h^{\mathrm{prtw}}=20\right)$, but much less by the compressive twins $\left(h^{\mathrm{prctw}}=2\right)$. This latter result may indicate either that prismatic dislocations can punch more easily through the compressive twin interface, or that tensile twin barriers are more closely spaced in the grains than compressive twin barriers.

The tensile and compressive stress-strain curves
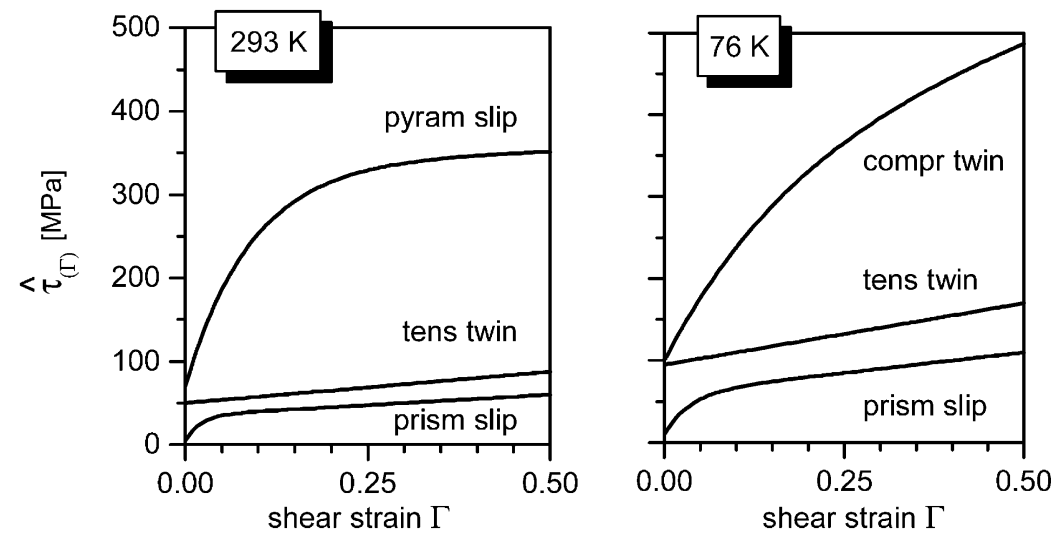

Fig. 5. Reference hardening of individual systems [equation (4)] adjusted to the experimental curves of Fig. 
Table 1. Parameters describing the evolution of threshold stress with deformation [Equations (4) and (5)] for the deformation modes and temperatures considered in this work

\begin{tabular}{|c|c|c|c|c|c|c|c|c|c|c|}
\hline \multirow[t]{2}{*}{ Temperature } & \multirow[t]{2}{*}{ System $(s)$} & \multirow[t]{2}{*}{$\tau_{0}(\mathrm{MPa})$} & \multirow[t]{2}{*}{$\tau_{1}(\mathrm{MPa})$} & \multirow[t]{2}{*}{$\theta_{0}(\mathrm{MPa})$} & \multirow[t]{2}{*}{$\theta_{1}(\mathrm{MPa})$} & \multirow{2}{*}{$\begin{array}{l}\text { Self-hard } \\
h^{s s}\end{array}$} & \multicolumn{4}{|c|}{ Latent hardening } \\
\hline & & & & & & & $h^{s p r}$ & $h^{s p y r}$ & $h^{s t t w}$ & $h^{s \mathrm{ctw}}$ \\
\hline \multirow[t]{3}{*}{ RT } & $\operatorname{Pr}$ & 5 & 30 & 1500 & 50 & 1 & 1 & 1 & 10 & - \\
\hline & Pyr & 70 & 270 & 3000 & 25 & 1 & 1 & 1 & 10 & - \\
\hline & Ttw & 50 & 0 & 75 & 75 & 1 & 1 & 1 & 10 & - \\
\hline \multirow[t]{3}{*}{$\mathrm{LN}$} & $\operatorname{Pr}$ & 10 & 50 & 1500 & 100 & 1 & 1 & - & 20 & 2 \\
\hline & Ttw & 95 & 0 & 150 & 150 & 1 & 1 & - & 10 & 10 \\
\hline & Ctw & 100 & 250 & 1700 & 300 & 1 & 1 & - & 10 & 10 \\
\hline
\end{tabular}

predicted using the hardening parameters listed in Table 1 are depicted in Fig. 2, superimposed with the experimental measurements. It can be seen that the model captures the major hardening features of these curves and specifically the increase in hardening rate, which is characteristic of twinning activity and appears only after some deformation has accumulated. Another feature entirely related to twinning is the deviation of IPC from IPT both, at 293 and 76K. As we will see in what follows, during IPT deformation is entirely accommodated by prismatic slip, while tensile twins activated during IPC induce a larger hardening.

\subsection{Texture evolution and activity of deformation systems}

The stress-strain evolution is only one aspect of the mechanical response. A sound constitutive model should also capture other features of deformation, such as: texture development, deformation mode activity and bulk anisotropic deformation. We will discuss these aspects in what follows. A qualitative understanding can be achieved if one considers the characteristics of the initial texture and that the $c$-axis represents the hard direction in $\mathrm{Zr}$ single crystals. As a consequence, enforcing deformation along the ND of the sheet requires the activation of the much harder compressive twinning (at LNT) or pyramidal slip (at RT). This explains the higher yield in the case of TTC, both at RT and LNT. In addition, when analyzing the predicted activity (Fig. 6), it can be seen that pyramidal slip dominates the deformation at RT, while compressive twins play an important role at LNT. In the latter case the predicted twinned fraction increases rapidly at the beginning and grows to about $70 \%$ of the total volume after $24 \%$ compressive deformation.

In the case of IPC or IPT, the sample can deform within the plane of the sheet, without having to change dimension along the harder ND. As a consequence, nearly plane strain deformation takes place via prismatic activity (Fig. 6) and the sample develops an ellipsoidal section. At this point it is interesting to compare the measured cross-section after TTC and IPC tests with the results of the simulations done with the coupled EPIC-VPSC code that will be described in next section (Fig. 7). This case was used to bench- mark the interface between VPSC and the FE code EPIC, since deformation is uniform in the compression sample and the result of the FE calculation should coincide with the prediction of the polycrystal code. In the TTC case, compression takes place along the axis of symmetry of the sample, which remains cylindrical in section (Fig. $7 \mathrm{~b}$ and c).

In what concerns in-plane testing, for the IPC case at RT the in-plane expansion is $\varepsilon_{22}=22 \%$ and the out-of-plane expansion is $\varepsilon_{33}=6 \%$ for a compressive strain of $\varepsilon_{11}=-28 \%$. Figure $7 \mathrm{~d}$ and e show that the simulated sections accurately coincide with the experimental ones [19] for the RT tests. In the case of IPC, the deviatoric component of stress along the ND is large enough to activate some tensile twinning both at RT and LNT. The reorientation associated with such twinning tends to "spread" the basal texture component along the ND and make the sample more "isotropic". As a consequence, although there is still ovalization of the sample, the latter is not so severe (see LN IPC in Fig. 3a). As for the associated simulations, we do predict tensile twinning activation (Fig. 6) for IPC, but our predictions tend to overemphasize the ovalization of the sample. In the case of IPT, on the other hand, the compressive deviatoric component along the ND is not large enough to activate either pyramidal slip at RT or compressive twinning at LNT. As a consequence, and since tensile twinning cannot be activated, deformation is overwhelmingly accommodated by plane strain via prismatic slip (Fig. 6 ) and the samples develop an extreme ovalization both at LNT (Fig. 3b) and at RT. In this case the constitutive model correctly captures such behavior.

The history of slip and twinning activity determines the texture evolution in the deformed samples (Fig. 8). Since prismatic slip activity does not reorient the $c$-axis, the basal pole figures after IPT are nearly identical to the initial texture. The activation of tensile twins during IPC causes a nearly $90^{\circ}$ reorientation of the $c$-axis along the compressive direction. The formation of such component is evident in Fig. 8. As for the textures associated with TTC, at RT the pyramidal activity rotates the $c$-axes away from the compressive loading axis, while at LNT the compressive twin reorientation creates a fiber perpendicular to the compressive axis.

Experimental textures obtained by OIM are avail- 

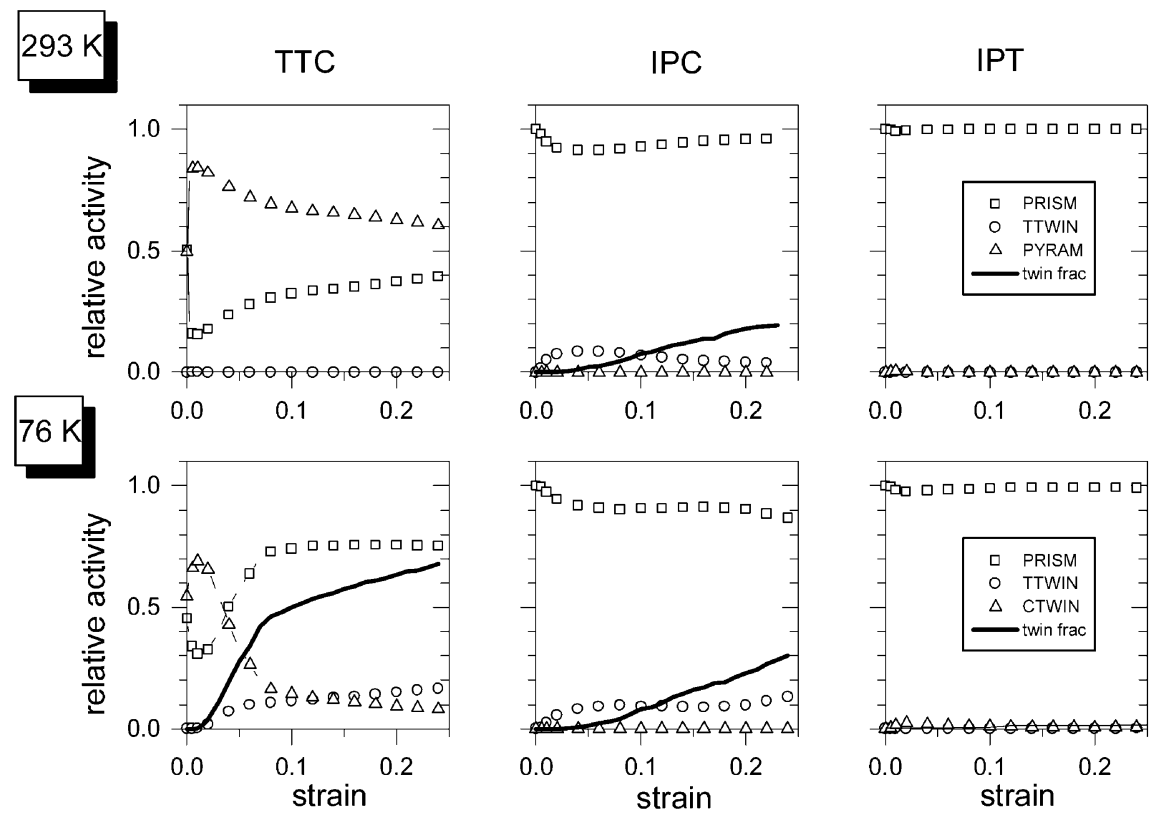

Fig. 6. Evolution of the relative activity of deformation modes and twin fractions with deformation, for TTC, IPC and IPT simulations.

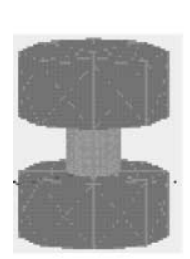

(a)

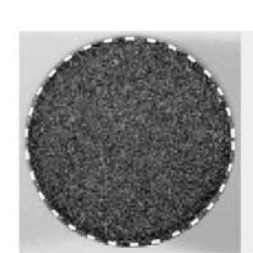

(b)

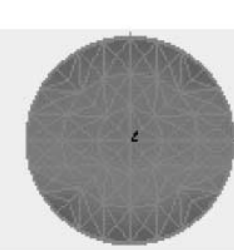

(c)

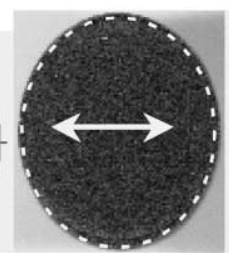

(d)

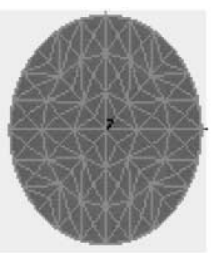

(e)

Fig. 7. Comparison of final sections of the $\mathrm{Zr}$ compression samples tested at 293K: (a) initial EPIC FE mesh of the uniaxial compression test; (b) photograph of the TTC experimental shape after $28 \%$ compression; and (c) the calculated TTC shape using EPIC; (d) photograph of the IPC experimental shape after $28 \%$ compression; and (e) the calculated IPC FE shape. For both (b) and (d) the outer circumference of the calculated shape (white dashed curve) has been superimposed over the experimental shape.

able for the case of TTC and IPT at 76K, and we compare them with the predicted textures in Fig. 9. Except for the difference in intensity, which is systematically larger for predicted textures, the comparison supports the predicted contribution to deformation of the various deformation modes. The basal pole figures for TTC are consistent with strong reorientation by compressive twinning. The pole figures for IPT, on the other hand, are consistent with predominant prismatic slip which tends to leave the basal poles invariant while aligning the $\{10 \overline{1} 0\}$ planes with the tensile axis.

Figure 10 shows the evolution of the predicted polycrystal yield surface (PCYS) during TTC, IPC and IPT deformation at LNT. These surfaces of equal work-rate are generated by stopping the simulation at regular deformation intervals and probing the aggregate, accounting for the actual texture, hardening, and grain shape at such deformation. A feature common to the three cases is the non-centro-symmetry of the yield surface, associated with the directionality of twinning. For the IPT case deformation is accommodated via prismatic slip and there is no change in the orientation of the $c$-axes. As a consequence, hardening is nearly proportional. In the case of TTC, the strong reorientation of the $c$-axes by twinning induces an important evolution with deformation of the shape of the PCYS, while the effect of twinning on the evolution of the PCYS associated with IPC is not as marked. Figure 10 illustrates the necessity of accounting for texture evolution effects for this class of materials.

\section{SIMULATION OF BEAM BENDING}

One of the reasons for developing the polycrystal constitutive equation described above is to be able to simulate complex forming operations of $\mathrm{Zr}$ with the help of FE techniques. The interfacing of polycrystal and FE codes is a rather novel approach which offers 


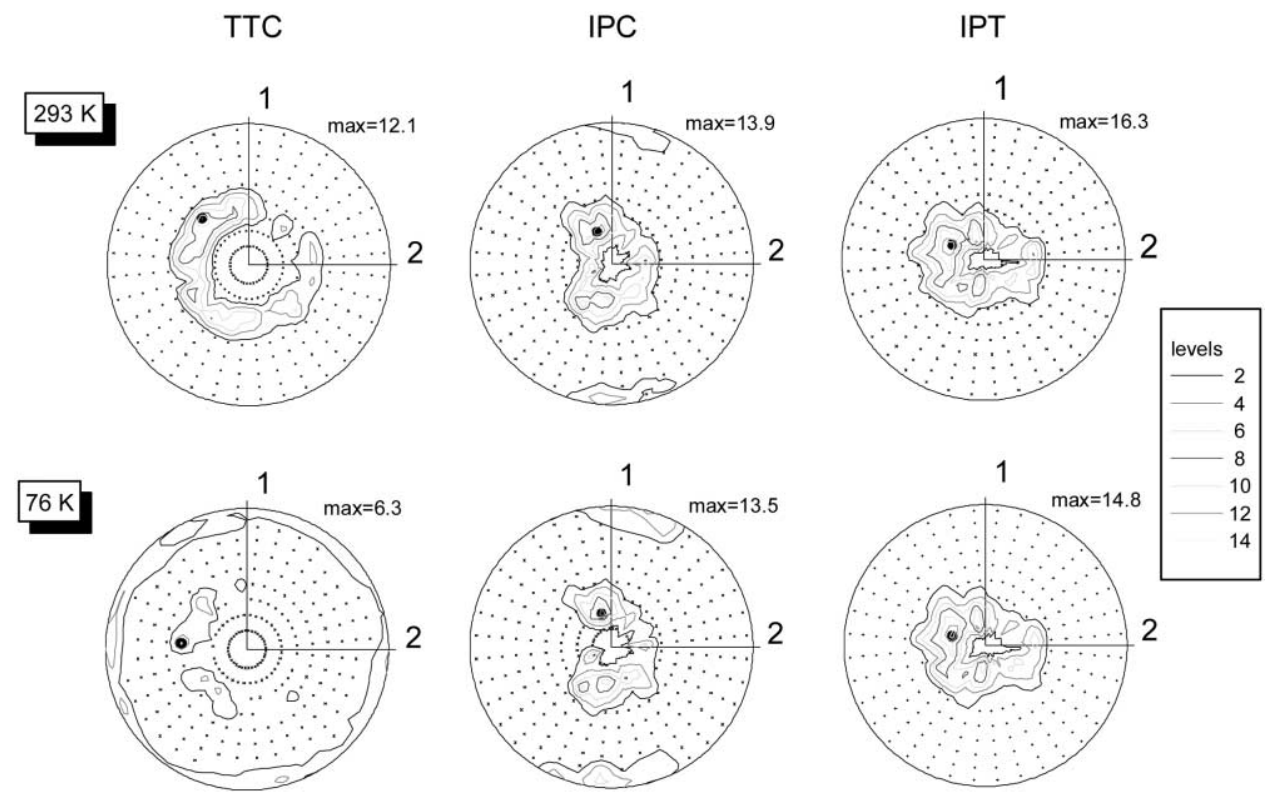

Fig. 8. Basal pole distributions predicted after 24\% TTC, IPC and IPT simulations done at 293 and 76K.

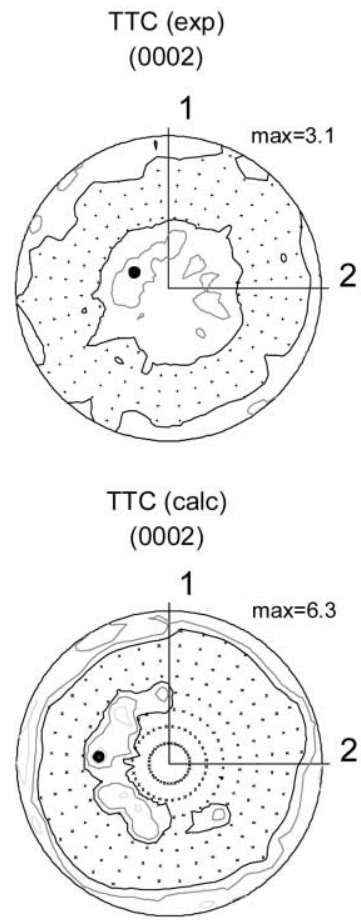

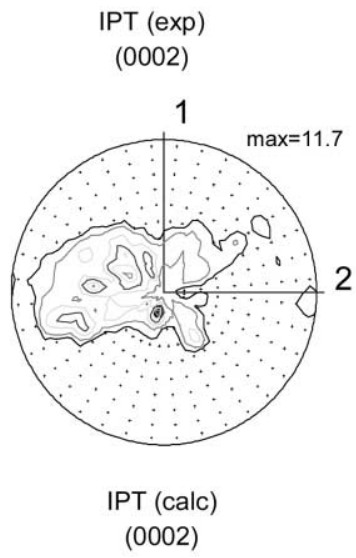
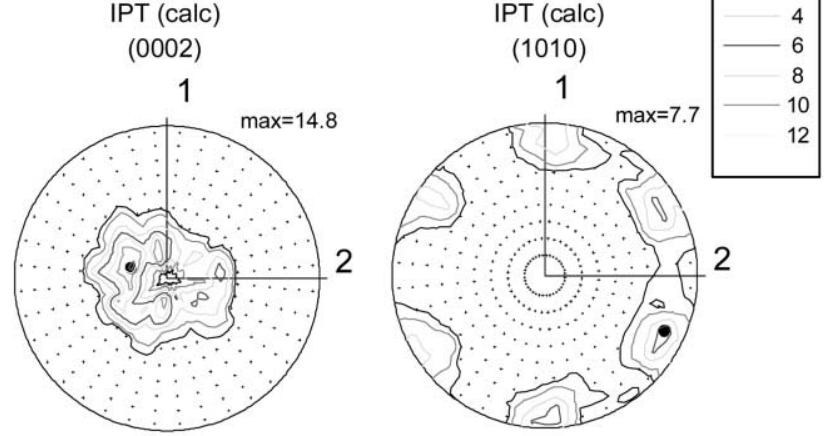

Fig. 9. Comparison of predicted and experimental textures after $24 \%$ TTC and IPT at $76 \mathrm{~K}$.

the possibility of accounting for the anisotropic evolution of the plastic properties of the aggregate, such as the TTC case illustrated in Fig. 10. So far it has being applied mainly to cubic materials [20-24] and, to the authors' knowledge, twinning has been incorporated in only one of such calculations [24].

The constitutive response developed above will be used in this Section to simulate a four-point bend beam test performed on rectangular bars of square section cut from the clock-rolled $\mathrm{Zr}$ described in the previous sections. The reason for choosing the bend beam test is because it provides non-homogeneous forming conditions with deformation gradients and local variables which are amenable to experimental analysis. In Part II [25] we present a detailed comparison of simulation and experiments for beams tested in bending at RT and LNT, with the main texture component (along the ND) contained in the bend- 
TTC

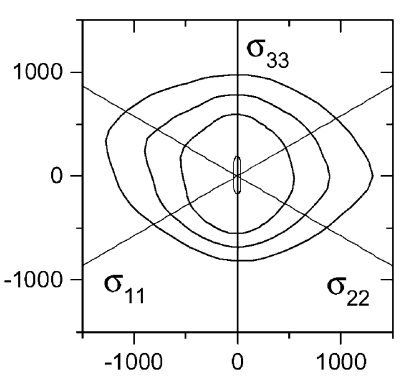

IPC

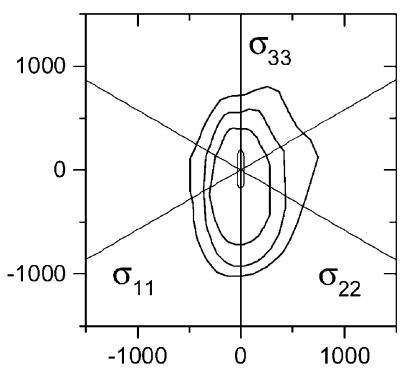

IPT

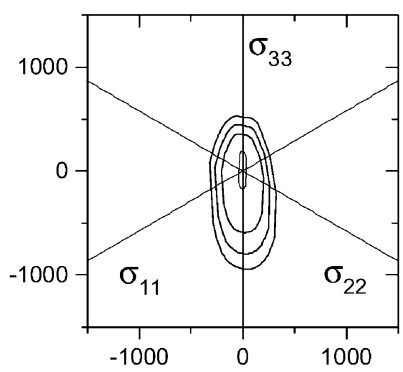

Fig. 10. PCYSs ( $\pi$-plane representation) associated with evolution of texture and hardening during TTC, IPC and IPT at $76 \mathrm{~K}$. Yield surfaces correspond to $0,8,16$ and $24 \%$ deformation.

ing plane ( $\mathrm{C} 0$ case) or perpendicular to it ( $\mathrm{C} 90$ case). In this paper we will describe the procedure for implementing the polycrystal code VPSC into the FE code EPIC, and provide preliminary simulation results that illustrate the role of the constitutive equation in the predicted response.

\subsection{Interfacing VPSC with EPIC}

In principle the interface between EPIC-97 [6] and the polycrystal code VPSC could be very simple: EPIC could merely interrogate VPSC from every FE integration point at each time cycle when an update of the deviatoric stress tensor is required for solution of the conservation equations. However, the computational cost of this approach would be prohibitive for an explicit dynamics code engaged in three-dimensional calculations. For a spatially well-resolved application problem the Courant-Friedrichs-Lewy controlled time step is typically on the order of $10 \mathrm{~ns}$ which implies a strain increment of about $10^{-5}$ for an sample strain rate of $10^{3} \mathrm{~s}^{-1}$. As a consequence, a feasible alternative is to interrogate VPSC and to update the materials properties at a user specified von Mises strain increment, $\delta \varepsilon_{\mathrm{Vm}}$ :

$$
\delta \varepsilon_{\mathrm{vM}} \equiv \int_{t_{m-1}}^{t_{m}}\left(\frac{2}{3} \bar{D}: \bar{D}\right)^{1 / 2} \mathrm{~d} t
$$

where the interval $\Delta t_{m}=\left(t_{m}-t_{m-1}\right)$ represents the time increment needed to accumulate a user specified strain increment $\delta \varepsilon_{\text {user }}$ at some spatial integration point and contains many EPIC time cycles (typically ranges from 100 to 1000 cycles); the index " $m$ " in equation (9) designates a VPSC interrogation time. Once the criterion $\delta \varepsilon_{\mathrm{vM}} \geq \delta \varepsilon_{\text {user }}$ has been satisfied, then VPSC is called from EPIC with an averaged deformation-rate tensor given by:

$$
\hat{D}_{m}=\frac{1}{\Delta t_{m}} \int_{t_{m-1}}^{t_{m}} \bar{D} \mathrm{~d} t=\frac{\delta \bar{\varepsilon}^{m}}{\Delta t_{m}}
$$

and the VPSC interrogation index is advanced.

Using the above example of a strain rate of $10^{3} \mathrm{~s}^{-1}$ and specifying $\delta \varepsilon_{\text {user }}$ to be $2 \%$ implies that a VPSC stress interrogation occurs every $\approx 2000$ time cycles in EPIC. However, since EPIC requires a stress state every time cycle between VPSC interrogations, a simple extrapolation scheme can be employed as follows. First we define the VPSC interrogation in functional form as:

$$
\bar{\sigma}^{\prime}=\Phi(\bar{\varepsilon})
$$

where $\bar{\varepsilon}$ is a macroscopic strain measure. Next we expand the second order tensor function $\Phi$ in a Taylor series about the strain $\bar{\varepsilon}\left(t_{m}\right)$ :

$$
\begin{gathered}
\Phi(t)=\Phi\left(t_{m}\right)+\left.\frac{\partial \Phi}{\partial \bar{\varepsilon}}\right|_{t_{m}}: \delta \bar{\varepsilon}+\frac{1}{2} \delta \bar{\varepsilon}:\left.\frac{\partial^{2} \Phi}{\partial \bar{\varepsilon}^{2}}\right|_{t_{m}} \\
: \delta \bar{\varepsilon}+\frac{1}{6} \delta \bar{\varepsilon}:\left.\frac{\partial^{3} \Phi}{\partial \delta \bar{\varepsilon}^{3}}\right|_{t_{m}}: \delta \bar{\varepsilon}: \delta \bar{\varepsilon}+\ldots
\end{gathered}
$$

and assume that a linear extrapolation is sufficient for our purpose of estimating the stress state at times between $t_{m}$ and $t_{m+1}$, thus giving the simple form:

$$
\Phi(t) \cong \Phi\left(t_{m}\right)+\left.\frac{\partial \Phi}{\partial \varepsilon}\right|_{t_{m}}: \delta \bar{\varepsilon} \Rightarrow \sigma^{\prime}=\bar{\sigma}^{\prime m}+P^{m}: \delta \bar{\varepsilon}
$$

The plastic stiffness tensor $P^{m}$ appearing in equation (13) is estimated using a backward difference in terms of the VPSC stress states at times $t_{m}$ and $t_{m-1}$, and the associated average strain increment for the time increment $\Delta t_{m}$ :

$$
P_{i j k l}^{m} \equiv \frac{\left(\bar{\sigma}_{i j}^{\prime m}-\bar{\sigma}_{i j}^{\prime m-1}\right)}{\delta \varepsilon_{k l}^{m}} .
$$

Since $P^{m}$ has diagonal symmetry and since $\bar{\sigma}^{\prime}$ and $\bar{\varepsilon}$ are deviatoric, $P^{m}$ has a maximum of 15 independent coefficients to extrapolate the stress state for a general 
material. Assumptions of material and test symmetry would of course reduce the number of non-zero independent coefficients contained in $P^{m}$. In terms of the homogeneous effective medium response represented by the compliance form of equation (2), equation (14) can be rewritten as:

$$
P_{i j k l}^{m}=\frac{\left(M_{m, i j p q}^{-1} \bar{D}_{p q}^{m}-M_{m-1, i j p q}^{-1} \bar{D}_{p q}^{m-1}\right)}{\delta \varepsilon_{k l}^{m}} .
$$

The use of equation (13) to update the stress between VPSC interrogations was found to be numerically satisfactory for the bent beam problem discussed below. The discontinuous "jumps" in stress at time $t_{m+1}$ when comparing FE extrapolated stresses via equation (13) to stand-alone VPSC calculations were rather small for a $\delta \varepsilon_{\text {user }}$ of $2 \%$. The VPSC runs were done at smaller strain increments of $0.5 \%$. The application of equation (13) to dynamic problems that involve local stress wave reverberations of significant amplitude and changing sign could be numerically problematic.

\subsection{Bent beam application}

In this section we describe the FE simulations of the bent beam tests and compare the predicted and measured final beam sections. Local textures and local strains are discussed in Part II [25]. For all simulation cases the initial texture of clock-rolled $\mathrm{Zr}$ (Fig. 1) and the active modes and hardening laws corresponding to LNT and RT from Table 1 are used. For comparison purposes results obtained with an isotropic continuum approach (von Mises) and a full constrained (Taylor) polycrystal approach are reported, in addition to the results of the VPSC polycrystal constitutive law. For each response we considered two orientations of the main basal component of the $\mathrm{Zr}$ texture: a case with the ND of the plate contained in the bending plane $(\mathrm{C} 0)$ and a second case where the ND is perpendicular to it (C90). In both cases the ND is perpendicular to the beam axis. In addition, we considered two deformation temperatures (LNT and RT). Thus, a total four bending cases were investigated and are designated here as $\mathrm{LNC} 0$, LNC90, RTC0 and RTC90.

Since the ND represents the hard direction in this material, we expect small deformation along the ND and, as a consequence, the beam cross-sections to be quite different in geometric shape depending on the position of the ND with respect to the bending plane. A comparison between the Taylor and the self-consistent results should indicate the relevance of enforcing at least five active systems per grain versus accommodation of the deformation with less than five systems. We expect local plane-strain deformation for the self-consistent calculation via activity of mainly prismatic slip, and a harder response from the fully constrained approach via forced activity of pyramidal and twinning systems.

Three-dimensional EPIC-97 FE simulations of the
$\mathrm{Zr}$ bent beam tests were performed with coupled polycrystal plasticity as described in Section 4.1, and starting from the undeformed version of the mesh shown in Fig. 11 (Fig. 11 portrays the final bent beam configuration). This beam was initially $50.8 \mathrm{~mm}$ long with a square cross-section of $6.35 \mathrm{~mm}$. Free-surface boundary conditions for the beam are imposed in the FE simulations except at the contact points of the four pins (upper pin contacts are located at $y= \pm 6.35 \mathrm{~mm}$ and lower contacts are located at $y= \pm 12.7$. The beam bends as the upper pins are displaced down by $u_{z}=-6 \mathrm{~mm}$ and the lower pins are held rigid. Although the EPIC calculations were performed at strain-rates of roughly $10^{3} \mathrm{~s}^{-1}$, the predicted deformation and dimensional changes of the beam are independent of the precise strain-rate imposed. The mesh shown in Fig. 11 contains 1920 tetrahedral (single-integration-point) elements with the tetrahedra symmetrically arranged in set of 24 [6] per hexahedron apparent in the figure. In order to conserve elements this mesh uses two symmetry planes that are coincident with the $x-z$ and $y-z$ planes. The use of these symmetry planes is justified if the initial texture is strictly orthotropic, while in our case we disregard the slight asymmetry displayed by the clock-rolled Zr plate (Fig. 1). Approximately 16000 time cycles were required to bend the beam into the final configuration shown in Fig. 11, producing von Mises equivalent strains of around $20 \%$ in the upper and lower fibers. Texture and hardening updates were conducted in the EPIC/VPSC interface at increments of $2.0 \%$ von Mises strain as discussed in Section 4.1, with an initialization ramp from 0 to $1 \%$ strain that used much smaller increments. The latter is done to account for the rapid initial hardening of the material and the fact that we rely on a forward extrapolation for hardening and stress updates [equation (13)].

Figure $12 \mathrm{a}$ presents a calculated $x-z$ cross-section of the bent beam for the simple constitutive response of an isotropic von Mises yield surface evolving according to a mechanical threshold strength hardening equation [26] adjusted to the ND response of the original $\mathrm{Zr}$ plate. The wedge-shaped section shown in Fig. 12a results from a near uniaxial stress loading along the beam axis ( $y$-axis) that reverses sign at the neutral plane in conjunction with the principle of

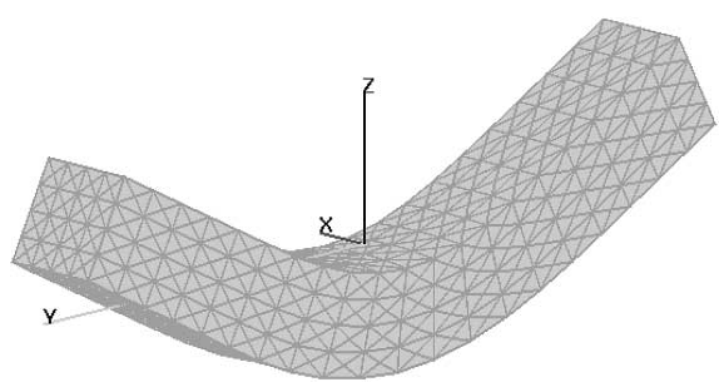

Fig. 11. Final configuration of the FE mesh for the bend bar specimen. 

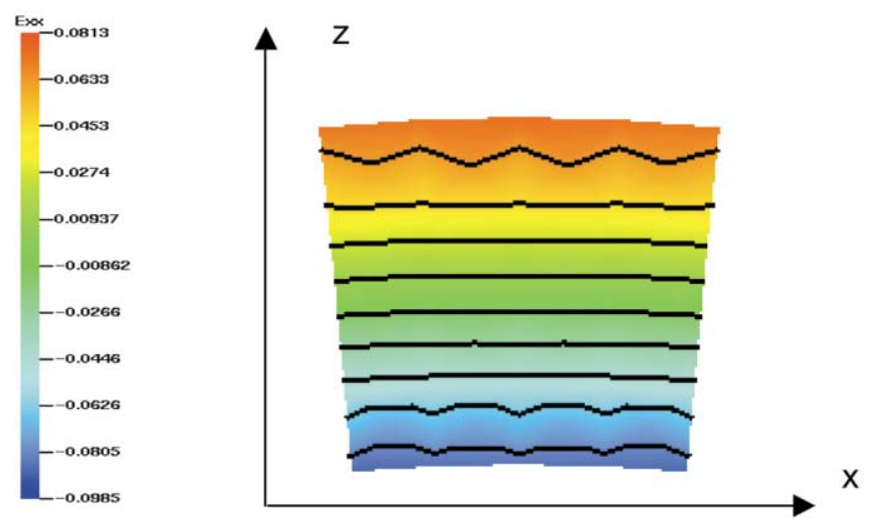

(a)

(b)
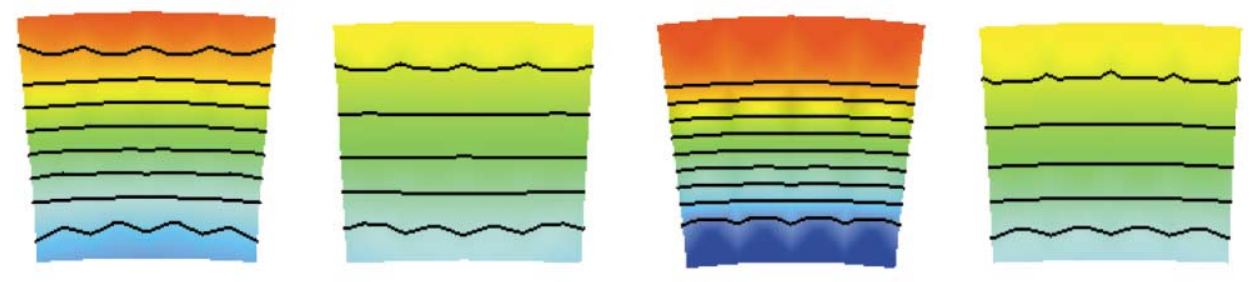

(c)

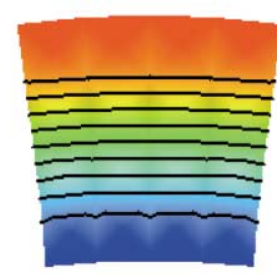

(LNCO)

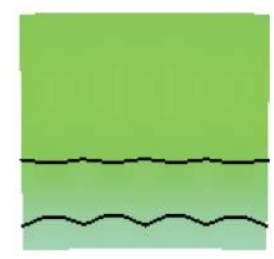

(LNC90)

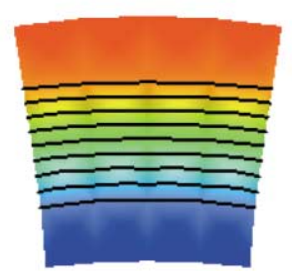

(RTCO)

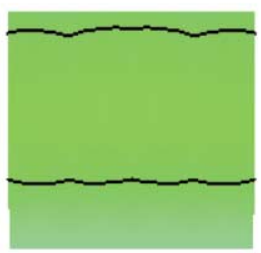

(RTC90)

Fig. 12. Final configuration for the $x-z$ section of the bend bar and contours of $E_{x x}^{p}$ calculated using (a) a Von Mises isotropic law, (b) a fully constrained Taylor and (c) a self-consistent polycrystal plasticity constitutive response for ND contained in (C0) and perpendicular to (C90) the bending plane, and for LN and RT.

plastic incompressibility. Uniaxial compression is realized above the neutral plane and uniaxial tension is realized below. Lagrangian strain along the $x$-direction is portrayed in Fig. 12 as contour lines that range (top to bottom) from $E_{x x}^{p}=-10 \%$ to $+8 \%$ for this beam deformation.

Figure 12 also presents calculated $x-z$ cross-sections of the bent beam for the constitutive response of a Taylor fully constrained polycrystal (Fig. 12b) and of a self-consistent polycrystal (Fig. 12c). Twinning, texture and hardening evolution are accounted for in both cases as described above in Section 3. The wedge-shaped sections shown in Fig. 12b and c correspond, from left to right, to the cases $\mathrm{LNC}$, LNC90, RTC0 and RTC90, respectively. Note that all four Taylor cases show wedged cross-sections, with the $\mathrm{C} 90$ cases less so ( $c$-axes are mostly perpendicular to the bending plane) and the $\mathrm{C} 0$ cases more so ( $c$ axes are mostly contained in the bending plane). Fig- ure $12 \mathrm{~b}$ indicates that less plastic strain in the $x$-direction is realized for the $\mathrm{C} 90$ cases versus the $\mathrm{C} 0$ cases (say $3 \%$ versus $12 \%$ ) reflecting that the $c$-component along the ND represents the "hard" direction in this material. As for Fig. 12c, note that only the $\mathrm{C} 0$ cases show wedged geometry, with the C90 cases producing rectangular geometry and vanishing $E_{x x}^{p}$ strain in the "hard" direction. A comparison of the calculated self-consistent $x-z$ cross-sections from Fig. 12c with experimental sections is done in Part II [25], where we show that the Taylor calculations overpredict the "wedging" effect for the $\mathrm{C} 90$ beams. The self-consistent approach, on the other hand, predicts final beam sections in better agreement with the experimentally measured ones.

\section{CONCLUSIONS}

Describing the constitutive response of anisotropic materials such as the clock-rolled $\mathrm{Zr}$ studied in this 
paper requires to account not only for the initial anisotropy and hardening, but for their evolution with strain as well. Here we show that a self-consistent polycrystal model (which features hardening and texture evolution) captures the constitutive response of textured $\mathrm{Zr}$. This crystallographic characterization has the advantage, as opposed to a constitutive formulation based on continuum mechanics, of being independent of the deformation path imposed on the aggregate. The polycrystal approach requires the active crystallographic mechanisms to be identified and the associated hardening parameters to be adjusted using extensive experimental information. We show that the mechanical response of textured $\mathrm{Zr}$ is the result of a complex interaction between the active deformation modes, and that accounting for anisotropic hardening is as important as accounting for texture evolution when adjusting the constitutive response.

In what concerns the twinning model, the predominant twin reorientation scheme used here is primarily meant to describe twinning contribution to texture development. The twinning contribution to hardening is treated here using a very simple approach. Namely, the barriers that twin lamellae pose to propagation of other twin and slip systems are accounted for by means of a latent hardening parameter. A more specialized model proposed recently by Karaman et al. [27] to describe deformation of Hadfield steel uses an extra term in the hardening law. This term, which provides an improved representation of the twinning process, depends on the evolving twin fraction and the separation between twin lamellae in the grain.

We show in this work that it is feasible to introduce the polycrystal response in a FE code, in order to describe complex forming operations. As an example, we present a preliminary simulation of bend beam tests which predicts very different outcomes depending on temperature and the relative orientation of texture and bending plane. A detailed comparison with actual experiments, including texture analysis, twinning statistics and local deformation measurements is presented in a companion paper [25].

Acknowledgements-This work was supported by the United States Department of Energy. Part of it was done under the auspices of Office of Basic Energy Sciences, Division of Materials Sciences.

\section{REFERENCES}

1. Lebensohn, R. A. and Tomé, C. N., Acta metall. mater. 1993, 41, 2611.
2. Lebensohn, R. A. and Tomé, C. N., Mater. Sci. Engng, 1994, A175, 71.

3. Castelnau, O., Duval, P., Lebensohn, R. A. and Canova, G. R., J. Geophys. Res., 1996, B101, 13851.

4. Lebensohn, R. A., Wenk, H. -R. and Tomé, C. N., Acta mater., 1998, 46, 2683.

5. Takeshita, T., Wenk, H. -R. and Lebensohn, R. A., Tectonophysics, 1999, 312, 133.

6. Johnson, G. R., Stryk, R. A., Holmquist, T. J. and Beissel. S. R., User Instructions for the 1997 Version of the EPIC Code. Wright Laboratory, Armament Directorate, Eglin Air Force Base Report, WL-TR-1997-7037, 1997.

7. Kaschner, G. C., Gray, G. T. III and Chen, S. R., in Proc. of Topical Conf. on Shock Compression of Condensed Matter, ed. D. P. Schmidt, D. P. Dandekar and J. W. Forbes. American Institute of Physics, Amherst, MA, 1997, p. 435.

8. Kaschner, G. C. and Gray, G. T. III, Metall. Mater. Trans., 2000, 31A, 1997.

9. Molinari, A., Canova, G. R. and Ahzi, S., Acta metall., 1987, 35, 2983.

10. Lebensohn, R. A., Turner, P. A., Signorelli, J. W., Canova, G. R. and Tomé, C. N., Mod. Sim. Mater. Sci. Engng, 1998, 6, 447.

11. Tomé, C. N., Mod. Sim. Mater. Sci. Engng, 1999, 7, 723.

12. Tomé, C. N., Lebensohn, R. A. and Kocks, U. F., Acta metall. mater., 1991, 39, 2667.

13. Reed-Hill, R. E., Deformation Twinning, ed. R. E. ReedHill, J. P. Hirth and H. C. Rogers. Gordon and Breach Science Publishers, New York, 1964, p. 295.

14. Tenckhoff, E., Metall. Trans., 1978, 9A, 1401.

15. Pochettino, A., Gannio, N., Vial, C. and Penelle, R., Scripta metall. mater., 1992, 27, 1952.

16. Lebensohn, R. A., Gonzalez, M. I., Pochettino, A. and Tomé, C. N., J. Nucl. Mater., 1996, 229, 57.

17. Lebensohn, R. A. and Tomé, C. N., Textures Microstruct., 1996, 26/27, 513.

18. Signorelli, J. W., Logé, R. E., Chastel, Y. B. and Lebensohn, R. A., Mod. Sim. Mater. Sci. Engng, 2000, 8, 193.

19. Maudlin, P. J., Tomé, C. N., Kaschner, G. C. and Gray, G. T. III, in Proc. 6th Int. Conf. on Numerical Methods in Industrial Forming Processes, NUMIFORM-98, ed. J. Huétink and F. P. T. Baaijens. Balkema, Rotterdam, 1998, p. 309.

20. Dawson, P. R., Beaudoin, A. J. and Mathur, K. K., in Proc. 4th Int. Conf. on Numerical Methods in Industrial Forming Processes, NUMIFORM-92, ed. J. L. Chenot, R. P. Wood and O. C. Zienkiewickz. Balkema, Rotterdam, 1992, p. 25.

21. Beaudoin, A. J., Dawson, P. R., Mathur, K. K. and Kocks, U. F., Int. J. Plasticity, 1995, 11, 501.

22. Anand, L., Kalidindi, S. R. and Bronkhorst, C. A., J. Mech. Phys. Solids, 1992, 40, 537.

23. Bronkhorst, C. A., Kalidindi, S. R. and Anand, L., Phil. Trans. R. Soc. London, 1992, A341, 443.

24. Kalidindi, S. R., J. Mech. Phys. Solids, 1998, 46, 267.

25. Kaschner, G. C., Bingert, J. F., Liu, C., Lovato, M., Maudlin, P. J., Stout, M. S. and Tomé, C. N., Acta mater., 2001, 49(15), 3097-3107.

26. Chen, S. R. and Gray, G. T. III, Phys. IV France, 1997, 7(C3), 741.

27. Karaman, I., Sehitoglu, H., Beaudoin, A. J., Chumlyakov, Y. I., Maier, H. J. and Tomé, C. N., Acta mater., 2000, 48, 2031. 